\title{
Basic Fibroblast Growth Factor Improves Myocardial Function in Chronically Ischemic Porcine Hearts
}

\author{
Kazumasa Harada, ${ }^{*}$ William Grossman, ${ }^{*}$ Menachem Friedman, ${ }^{\ddagger}$ Elazer R. Edelman," Pottumarthi V. Prasad, $\$$ \\ Craig S. Keighley, ${ }^{*}$ Warren J. Manning, ${ }^{*}$ Frank W. Sellke, ${ }^{\ddagger}$ and Michael Simons* \\ *The Charles A. Dana Research Institute and Harvard-Thorndike Laboratory, Department of Medicine, Cardiovascular Division, \\ ${ }^{\ddagger}$ Department of Surgery, Division of Cardiothoracic Surgery, and ${ }^{\S}$ Department of Radiology, Beth Israel Hospital; "Department of \\ Medicine, Cardiovascular Division, Brigham and Women's Hospital, and Harvard Medical School, Boston, Massachusetts 02115
}

\begin{abstract}
The effect of basic fibroblast growth factor (bFGF) administration on regional myocardial function and blood flow in chronically ischemic hearts was studied in 26 pigs instrumented with proximal circumflex coronary artery (LCX) ameroid constrictors. In 13 animals bFGF was administered extraluminally to the proximal left anterior descending (LAD) and LCX arteries with heparin-alginate beads and 13 other animal served as controls. bFGF-treated pigs showed a fourfold reduction in left ventricular infarct size compared to untreated controls (infarct size: $1.2 \pm 0.4 \%$ vs. $5.1 \pm 1.3 \%$ of $\mathrm{LV}$ mass, mean \pm SEM, $P$ $<0.05)$. Percent fractional shortening (\% FS) in the LCX area at rest was reduced compared with the LAD region in both bFGF and control pigs. However, there was better recovery in the LCX area after rapid pacing in bFGF-treated pigs (\% $\mathrm{FS}_{\mathrm{LCX}} / \% \mathrm{FS}_{\mathrm{LAD}}, 22.9 \pm 7.3 \% \rightarrow 30.5 \pm 8.5 \%, P<0.05$ vs. prepacing) than in controls $(16.0 \pm 7.8 \% \rightarrow 14.3 \pm 7.0 \%, P=N S)$. Furthermore, $L V$ end-diastolic pressure rise with rapid pacing was less in bFGF-treated than control pigs (pre-pacing; pacing; postpacing, $10 \pm 1 ; 17 \pm 3 ; 11 \pm 1 * \mathrm{mmHg}$ vs $10 \pm 1 ; 24 \pm 4 ; 15 \pm 1$ $\mathrm{mmHg}, * P<0.05$ vs. control). Coronary blood flow in the LCX territory (normalized for LAD flow) was also better during pacing in bFGF-treated pigs than in controls. Thus, periadventitial administration of bFGF in a gradual coronary occlusion model in pigs results in improvement of coronary flow and reduction in infarct size in the compromised territory as well as in prevention of pacing-induced hemodynamic deterioration. (J. Clin. Invest. 1994. 94:623-630.) Key words: basic fibroblast growth factor • regional myocardial function • chronic ischemia - coronary artery disease $\cdot$ angiogenesis
\end{abstract}

\section{Introduction}

Basic-fibroblast growth factor (bFGF) ${ }^{1}$ is an intensely mitogenic $(1-3)$ as well as angiogenic $(2,4-6)$ single-chain $16-\mathrm{kD}$

Address all correspondence to Michael Simons, M.D., Cardiovascular Division, Beth Israel Hospital, 330 Brookline Avenue, Boston, MA 02215.

Received for publication 18 October 1993 and in revised form 10 February 1994.

1. Abbreviations used in this paper: bFGF, basic fibroblast growth factor; EDSL, end-diastolic segment length; ESSL, end-systolic segment length; FS, fractional shortening; LAD, left anterior descending coronary artery; LCX, left circumflex coronary artery; LV, left ventricle; LVEDP, left ventricular end-diastolic pressure.

J. Clin. Invest.

(C) The American Society for Clinical Investigation, Inc.

0021-9738/94/08/0623/08 \$2.00

Volume 94, August 1994, 623-630 peptide widely distributed in blood vessels $(3,7)$, cardiac tissues $(8,9)$ and other organs. Recently, Yanagisawa-Miwa et al. (8) reported that intracoronary administration of bFGF during an acute myocardial infarction in dog model, resulted in reduction in the infarct size and an increase in the number of capillaries and arterioles in the treated territory. However, the canine coronary circulation has a well developed native collateral circulation capable of preventing infarctions after gradual coronary occlusion without any additional interventions (10). In contrast, human and porcine coronary circulations have a sparse collateral network (11) which may respond differently to bFGF-induced angiogenesis. With these considerations in mind we undertook this study in order to test the effectiveness of bFGF in preventing myocardial infarction and physiologic deterioration in a porcine model of gradual coronary occlusion model.

We used the ameroid constrictor technique described by Roth et al. (12) to produce gradual (2-4 wk) occlusion of the proximal circumflex coronary artery (LCX) in pigs. This method allows for the development of a chronically ischemic region of the left ventricular myocardium that does not possess a preexisting collateral blood supply (13-15). Using this technique, O'Konski et al. (16) demonstrated that after 3-4 wk of gradual coronary artery occlusion, pigs develop a significant increase in coronary collateral blood flow to the compromised area reaching almost same level as the flow in the normal area at rest. However, the territory supplied by the ameroid-constricted artery is clearly ischemic during exercise or pharmacological vasodilation (17). The nature of this limitation remains unclear and it has been variously ascribed to new collaterals possessing less smooth muscle than normal arterioles (12), having impaired function (18) or simply to an insufficient number of collaterals forming in the ischemic territory. In a previous study we have demonstrated in a similar model that bFGF administration restores endothelial function in the ameroid-compromised territory (19). In this investigation we set out to determine whether bFGF treatment also improves regional left ventricular function in chronically ischemic myocardium. To this end, we used periadventitial delivery of bFGF using heparin-alginate polymer (2, 20, 21). This method allows reliable and sustained delivery with first-order kinetics over 4-6 wk (21). Furthermore, recent studies using this approach demonstrated local arterial deposition with little systemic loss (20).

\section{Methods}

Production of collateral vessels. 28 Yorkshire pigs of either sex weighing $10-20 \mathrm{~kg}$ were anesthetized with intramuscular ketamine (10 $\mathrm{mg} / \mathrm{kg}$ ) and halothane inhalation anesthesia. By sterile technique, a left thoracotomy was performed through the fourth intercostal space during mechanical ventilation. The pericardium was opened, and an ameroid constrictor of either 2.77 or $3.0 \mathrm{~mm}$ internal diameter was placed around the proximal circumflex coronary artery. In the basic FGF group ( $n$ 
$=13$ ), basic fibroblast growth factor was administered extraluminally to both the proximal left anterior descending and circumflex arteries by applying 4 or 5 calcium alginate capsules encapsulating heparin-sepharose beads containing bFGF ( $1 \mu \mathrm{g}$ bFGF per bead, total dose 4-5 $\mu \mathrm{g} /$ animal) secured with a small amount of hemostatic surgical gauze (Surgicel ${ }^{\otimes}$; Johnson \& Johnson, New Brunswick, NJ). In the control group $(n=13)$, an ameroid constrictor was placed around the proximal circumflex artery without any heparin-alginate beads $(n=7)$ or with heparin-alginate beads not containing bFGF $(n=6)$. Before ameroid placement, regional blood flow was measured by the colored microsphere technique (22), as described below. In all animals, the thoracotomy was closed and antibiotics (Cefazolin; Marsham, Cherry Hill, NC) were injected intramuscularly. Thereafter, the pigs were allowed to recover for $5 \mathrm{~d}$ and then were transferred to a farm until they were required by the second part of the study. Experiments were performed 5-8 wk after ameroid constrictor placement because preliminary study by coronary angiography showed complete occlusion of the circumflex coronary artery after $5 \mathrm{wk}$ in nearly all animals.

Preparation of basic-FGF containing heparin-alginate beads. Calcium alginate beads provide a stable platform for bFGF because of enhanced retention of activity and storage time, and served as a means for the controlled release of bFGF to the vessels in vivo. As described previously $(2,21)$ heparin-sepharose beads (Pharmacia LKB, Piscataway, NJ) were sterilized under ultraviolet light for $30 \mathrm{~min}$ and then mixed with filter-sterilized sodium alginate $(1.2 \%$, wt/vol; Sigma Chemical Co., St. Louis, MO). The mixed slurry was dropped through a needle into a beaker containing a hardened solution of $\mathrm{CaCl}_{2}(1.5 \% \mathrm{wt} /$ $\mathrm{vol})$. Beads were formed instantaneously. Uniformly crosslinked capsule envelopes were obtained by incubating the capsules in the $\mathrm{CaCl}_{2}$ solution for 5 min under gentle mixing, and then for $10 \mathrm{~min}$ without mixing. The beads were washed three times with sterile water and stored in $0.9 \% \mathrm{NaCl} / \mathrm{mM} \mathrm{CaCl}{ }_{2}$ at $4^{\circ} \mathrm{C}$. Each capsule in its hydrated state contained $0.05 \mathrm{mg}$ heparin-sepharose, $0.18 \mathrm{mg}$ of alginate, and $11 \mathrm{mg}$ of water. For heparin-alginate capsule loading with $\mathrm{bFGF}$, eight capsules were incubated in $0.9 \% \mathrm{NaCl} / / \mathrm{mM} \mathrm{CaCl} / 2 / 0.05 \%$ gelatin with $10 \mu \mathrm{g}$ of b-FGF (recombinant human bFGF 147 amino acids; R\&D Systems, Inc., Minneapolis, $\mathrm{MN}$ ), for $16 \mathrm{~h}$ under gentle agitation at $4^{\circ} \mathrm{C}$. Previous studies $(20,21)$ have shown that under these conditions, $80 \%$ of bFGF in solution is absorbed into heparin-alginate capsules. Thus, $8 \mu \mathrm{g}$ of bFGF is absorbed into 8 capsules, resulting in incorporation of $1 \mu \mathrm{g}$ bFGF/capsule.

Experimental preparation. On the day of the study, the pigs were anesthetized with intramuscular ketamine $(10 \mathrm{mg} / \mathrm{kg})$ and then with intravenous $\alpha$-chloralose and urethane $(3.2 \mathrm{~g} \alpha$-chloralose and $20 \mathrm{~g}$ urethane in $100 \mathrm{ml}$ normal saline; $60 \mathrm{ml}$ initially and $10-15 \mathrm{ml}$ every 45-60 min as needed), intubated and mechanically ventilated. Twodimensional transthoracic echocardiography was performed to evaluate left ventricular global function. A median sternotomy was performed using an oscillating saw. Adhesions were divided sharply with the use of scissors and electrocautery. Pacing electrodes were secured to the left atrial appendage. An 8F high-fidelity catheter-tip micromanometer (Millar Instruments, Houston, TX) was inserted into the left ventricular cavity via the left ventricular apex. To assess regional wall motion, two pairs of ultrasonic crystals were implanted in the subendocardium parallel to the short axis of the left ventricle (Fig. 1). One pair was placed in the distribution of the left anterior descending artery (normal), and another pair was placed in the distribution of the left circumflex artery (either collateral dependent area or infarct area). Both pairs were on the same trans-axial plane of the left ventricle. After recording left ventricular pressure and segment length in both normal and collateral-dependent regions (Honeywell-Electronics for Medicine Research Recorder, Denver, $\mathrm{CO}$ ), rapid atrial pacing (1.8 times resting heart rate) was performed for $4 \mathrm{~min}$. Hemodynamic and functional parameters were recorded before, during, and after pacing. Regional myocardial blood flow measurements using the colored microsphere technique (details below) were performed before and during pacing. Percent fractional shortening was calculated as (EDSL-ESSL)/EDSL $\times 100$, where EDSL is end-diastolic segment length and ESSL is end-systolic segment length.

Subsequently, the heart was excised and after the myocardium was

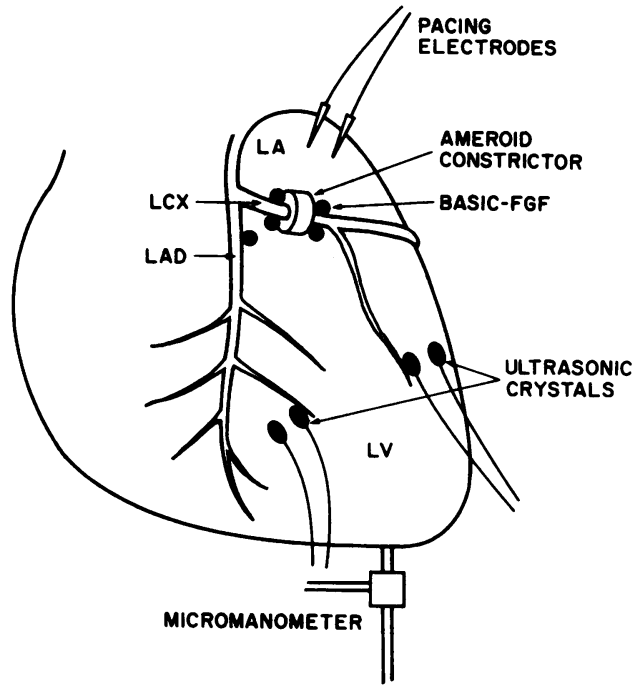

Figure 1. Animal preparation used in the present study. An ameroid constrictor was placed on the proximal LCX. bFGF was administered extraluminally to the proximal and distal LCX (2-3 capsules) and the proximal LAD ( 2 capsules) using heparin-alginate polymer. After 5 8 wk of recovery, LV pressure was measured with a micromanometer inserted through the left ventricular apex. Pairs of ultrasonic crystals were implanted in the area perfused by the LAD and the area perfused by the LCX.

sampled for regional myocardial blood flow measurement the remaining myocardium was fixed by immersion in phosphate-buffered $10 \%$ Formalin, $\mathrm{pH}$ 7.4. The area of the ameroid constrictor was examined visually, and circumflex coronary artery occlusion was confirmed. All animals received humane care in compliance with the Beth Israel Hospital Committee on Animal Research and the American Physiological Society.

Tissue analysis. After fixation for at least $2 \mathrm{~d}$, the left ventricle was sectioned into 6-8 transverse slices ( $\sim 1 \mathrm{~cm}$ thickness). Each slice was weighed, its apical surface was traced and the infarct area was determined visually by the scar. The volumes of the infarction were summed and divided by the volume of the left ventricle to yield the percent volume of the left ventricle which showed infarction. Anterior and posterior wall thickness were determined in multiple transverse sections by measuring endocardial to epicardial distances. Care was taken to determine myocardial wall thickness in the same plane and location in all specimens.

Transthoracic echocardiography. 5-8 wk after the ameroid placement, 2D transthoracic echocardiography was performed immediately after pigs were anesthetized with the animal in the left decubitous or spine position using an HP 1000 (Hewlett Packard Medical Systems, Andover, MA) equipped with $2.5 \mathrm{MHz}$ phased array transducer. Recording were made at the mid-papillary muscle level and stored on VHS tape for later analysis. 2D recordings were analyzed utilizing an offline computer analysis system (Cardiology Workstation; GTI Freeland Medical Division, Louisville, CO). Diastolic measurements (anterior, posterior wall thickness, end-diastolic dimension, end-diastolic area) were made coincide with the QRS complex on the ECG, while systolic measurements (end-systolic dimension, end-systolic area) were made at the point of minimum LV area. The mean of three consecutive beats was used for all measurements in each animal.

Myocardial blood flow measurement. We used the colored microsphere technique to quantify myocardial blood flow (22). In this technique, polystyrene spheres $(15 \pm 0.1[\mathrm{SD}] \mu \mathrm{m}$ in diameter) dyed with different colors are used in place of conventional radioactive microspheres. Flow can be quantified by analyzing the dye content using spectrophotometric methods (22). We used three sets of colored microspheres in each animal, one before the placement of the constrictor, one after maturation of the ischemic area (baseline) and one during pacing 

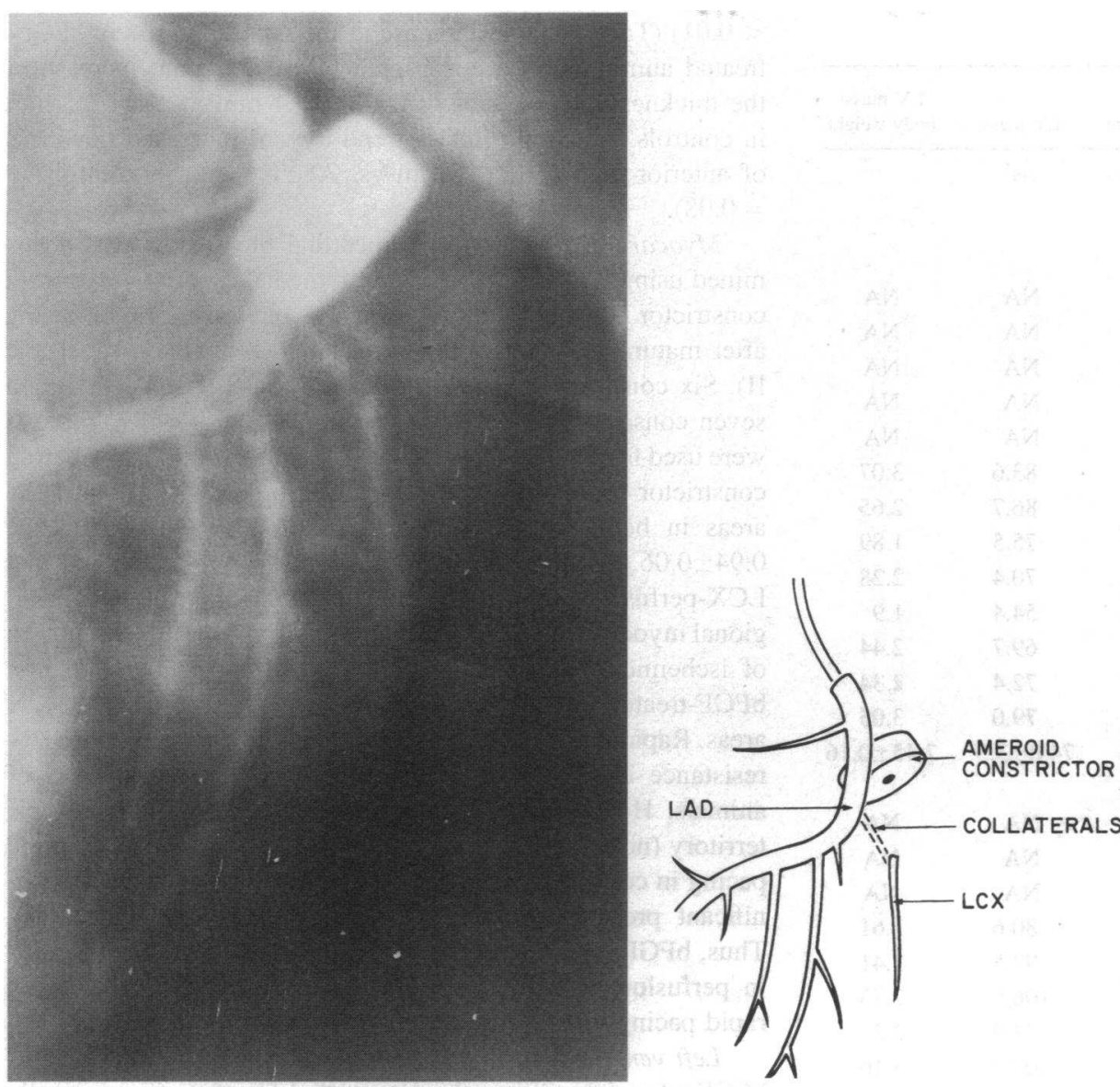

Figure 2. Coronary angiography performed $5 \mathrm{wk}$ after the placement of the ameroid constrictor with basic FGF treatment. LAD and LCX were visualized with Iopamidol. Total occlusion of the proximal LCX by the ameroid constrictor and faint collateral vessels from the branches of the LAD are observed.

after maturation of the ischemic area. Since we have worked with an open-chest model, Dye-Trak colored microspheres (Triton Technology Inc., San Diego, CA) were injected directly into the left atrium. Reference blood samples were withdrawn using a syringe pump at a constant rate of $4 \mathrm{ml} / \mathrm{min}$ through the femoral artery. The number of microspheres injected were typically 3 million (before placement of constrictor) and 6 million (after maturation of the ischemic area). The latter number of microspheres was increased due to animals almost doubling (10-20 kg to $25-30 \mathrm{~kg}$ ) its size during the 5-8-wk interval from the first study. After excision of the heart, the left ventricle was dissected free and a trans-axial slice of $\sim 1 \mathrm{~cm}$ thick was cut. From this slice, eight radial samples were made which were further dissected into epi-, mid-, and subendocardial samples. The tissue samples and the reference blood samples were digested with potassium hydroxide and microspheres were reclaimed using a vacuum filter. The dyes from the microspheres were extracted using dimethyl-formamide: The dye sample was analyzed in a spectrophotometer (HP 8452 A; Hewlett Packard, Palo Alto, CA). Complete details of the methodology and analysis are available in reference 22. From the optical density (OD) measurements, the myocardial flow (expressed in $\mathrm{ml} \cdot \mathrm{min}^{-1} \mathrm{~g}^{-1}$ ) was calculated using the following expression:

Blood flow (tissue sample $\mathrm{X}$ )

$=\left[\right.$ Withdrawal rate $\left(\mathrm{ml} \cdot \mathrm{min}^{-1}\right) /$ weight (tissue sample $\left.\left.\mathrm{X}\right)(\mathrm{g})\right]$

$\times[\mathrm{OD}$ (tissue sample $\mathrm{X}) / \mathrm{OD}$ (reference blood sample) $]$.

Data analysis. Data are reported as mean \pm SEM. A two-tailed paired $t$ test was used for comparison of variables. A $P$ value of less than 0.05 was considered significant.

\section{Results}

26 of 28 animals survived the ameroid constrictor placement and heparin-alginate or sham beads implantation. Survival rate was the same for both bFGF treated and control groups (13/14 in bFGF treated vs. 13/14 in controls). Both deaths were sudden and occurred within the first $24 \mathrm{~h}$ after surgery. There were no infectious complications associated with placement of bFGF capsules. Of 13 control animals, 6 were implanted with heparinalginate capsules without bFGF and 7 were without any capsules. There was no difference in any parameter measured (infarct size, wall thickness, coronary flow, or left ventricular function) between the two control groups and both groups are combined for purposes of data presentation. Pigs were studied 5 to 8 weeks (mean, $6.5 \mathrm{wk}$ ) after initial surgery. The mean time to study was $6.4 \pm 0.3$ weeks in control and $6.5 \pm 0.2$ weeks in bFGF-treated animals.

Coronary angiography performed in four animals 5 wk after ameroid constrictor placement demonstrated complete occlusion in three animals (Fig. 2) with one pig showing a subtotal occlusion. The remaining 22 pigs were studied at least $6 \mathrm{wk}$ after ameroid constrictor implantation and visual inspection during repeat surgical procedure demonstrated complete occlusion in all animals.

Morphologic analysis. $5 \mathrm{wk}$ after implantation of ameroid constrictors, myocardial infarction was absent in 2 of 13 (15.4\%) control and in 4 of $13(30.8 \%)$ bFGF-treated animals. There was a trend toward smaller number of transmural infarcts in bFGF group ( 6 of 13 vs. 9 of 13) that did not reach statistical significance. However, bFGF-treated pigs showed a fourfold reduction in the total infarction mass when compared to the untreated controls $(1.2 \pm 0.4 \%$ vs. $5.1 \pm 1.3 \%$ of $\mathrm{LV}$ mass, $P$ $<0.05)$. The total $\mathrm{LV}$ mass was larger in bFGF-treated pigs than in control animals $\left(92.5 \pm 4.4 \mathrm{~cm}^{3}\right.$ vs. $74.0 \pm 3.5 \mathrm{~cm}^{3}, P$ 
Table I. Morphometric Data

\begin{tabular}{|c|c|c|c|c|c|}
\hline Animal No. & $\begin{array}{l}\text { Time to } \\
\text { study }\end{array}$ & $\begin{array}{c}\text { Type of } \\
\text { infarction }\end{array}$ & Infarct size & LV mass & $\begin{array}{l}\text { LV mass/ } \\
\text { body weight }\end{array}$ \\
\hline & $w k$ & & $\%$ LV mass & $\mathrm{cm}^{3}$ & \\
\hline \multicolumn{6}{|l|}{$\begin{array}{l}\text { Non-bFGF } \\
\text { (control) }\end{array}$} \\
\hline 1 & 5 & $\mathbf{N}$ & NA & NA & NA \\
\hline 2 & 8 & $\mathrm{~T}$ & NA & NA & NA \\
\hline 3 & 5 & $\mathrm{~T}$ & NA & NA & NA \\
\hline 4 & 7 & $\mathbf{T}$ & NA & NA & NA \\
\hline 5 & 5 & $\mathrm{~T}$ & NA & NA & NA \\
\hline 6 & 6 & $\mathrm{~T}$ & 8.63 & 83.6 & 3.07 \\
\hline 7 & 6 & $\mathbf{N}$ & 0 & 86.7 & 2.65 \\
\hline 8 & 8 & $\mathrm{~T}$ & 4.9 & 75.5 & 1.89 \\
\hline 9 & 6 & $S$ & 2.9 & 70.4 & 2.28 \\
\hline 10 & 6 & $T$ & 11.3 & 54.4 & 1.9 \\
\hline 11 & 7 & $\mathrm{~T}$ & 7.2 & 69.7 & 2.44 \\
\hline 12 & 7 & $S$ & 2.0 & 72.4 & 2.34 \\
\hline \multirow[t]{2}{*}{13} & 7 & $\mathrm{~T}$ & 4.0 & 79.0 & 3.05 \\
\hline & $6.4 \pm 0.3$ & & $5.1 \pm 1.3$ & $74.0 \pm 3.5$ & $2.45 \pm 0.16$ \\
\hline \multicolumn{6}{|c|}{ bFGF-treated } \\
\hline 1 & 6 & $\mathrm{~T}$ & NA & NA & NA \\
\hline 2 & 7 & $\mathbf{S}$ & NA & NA & NA \\
\hline 3 & 5 & $\mathbf{S}$ & NA & NA & NA \\
\hline 4 & 6 & $\mathbf{S}$ & 3.3 & 80.6 & 2.61 \\
\hline 5 & 6 & $\mathbf{N}$ & 0 & 97.5 & 3.41 \\
\hline 6 & 7 & $\mathbf{N}$ & 0 & 106.5 & 2.73 \\
\hline 7 & 6 & $\mathrm{~T}$ & 0.8 & 72.9 & 2.2 \\
\hline 8 & 7 & $\mathbf{N}$ & 0 & 107.5 & 3.16 \\
\hline 9 & 7 & $\mathrm{~T}$ & .4 & 68.5 & 2.25 \\
\hline 10 & 6 & $\mathbf{T}$ & 1.4 & 90.8 & 2.7 \\
\hline 11 & 6 & $\mathbf{T}$ & 2.3 & 103.8 & 2.97 \\
\hline 12 & 8 & $\mathbf{T}$ & 3.5 & 94.0 & 1.95 \\
\hline \multirow[t]{2}{*}{13} & 8 & $\mathbf{N}$ & 0 & 102.7 & 2.29 \\
\hline & $6.5 \pm 0.2$ & & $1.2 \pm 0.4^{*}$ & $92.5 \pm 4.4^{\ddagger}$ & $2.63 \pm 0.15$ \\
\hline
\end{tabular}

Averaged LV infarct size in non-bFGF pigs and in bFGF pigs. $N$, no infarction; $T$, transmural infarction; $S$, subendocardial infarction; $N A$, not available. Values given as mean $\pm \mathrm{SE}$. Values are compared with each other group by unpaired $t$ test. ${ }^{*} P<0.05,{ }^{\ddagger} P<0.01$.
$<0.01$ ) (Table I). This increase in the total LV mass in bFGFtreated animals was accompanied by a significant increase in the thickness of posterior wall $(8.1 \pm 0.4 \mathrm{~mm}$ vs. $6.4 \pm 0.5 \mathrm{~mm}$ in controls, $P<0.05$ ) and a trend toward increased thickness of anterior wall $(9.1 \pm 0.4 \mathrm{~mm}$ vs. $7.8 \pm 0.6 \mathrm{~mm}$ in controls, $P$ $=0.08$ ).

Myocardial blood flow. Myocardial blood flow was determined using colored microsphere immediately prior to ameroid constrictor placement and both at rest and during rapid pacing after maturation of the ischemic area (5-8 wk later, see Table II). Six consecutive control animals (Nos. 8-13, Table I) and seven consecutive bFGF-treated animals (Nos. 7-13, Table I) were used for coronary flow studies. Coronary blood flow before constrictor placement was comparable in both LAD and LCX areas in both groups (LCX flow/LAD flow; $0.83 \pm 0.11$ vs. $0.94 \pm 0.06$, controls vs. bFGF-treated, $P=\mathrm{NS}$ ) suggesting that LCX-perfused territory was equal in both sets of animals. Regional myocardial blood flow at rest determined after maturation of ischemic zone, did not show any difference between non bFGF-treated and bFGF-treated pigs in both LAD and the LCX areas. Rapid pacing resulted in the expected decline in coronary resistance in both LAD and LCX territories in both sets of animals. However, while myocardial blood flow in the LCX territory (normalized for the LAD flow) decreased during rapid pacing in control animals, bFGF-treated pigs demonstrated significant preservation of LCX territory perfusion (Table II). Thus, bFGF administration resulted in significant improvement in perfusion in the chronically ischemic myocardium during rapid pacing.

Left ventricular hemodynamics. To determine the effect of bFGF administration on the myocardial function of chronically ischemic animals, we analyzed left ventricular hemodynamics and segment length fractional shortening before, during, immediately, and 5 min after pacing in bFGF-treated as well as control animals. Three pigs in each group were excluded from the study because hemodynamically unstable fast spontaneous heart rate induced during animal preparation. Therefore, hemodynamic data were available from 10 animals in each group (Table III).

Percent fractional shortening ( $\%$ FS) in the LCX area (ameroid region) at rest was reduced compared with the $L A D$ region in both non-bFGF $(3.7 \pm 1.9 \%$ vs. $19.1 \pm 1.8 \%)$ and bFGF-treated pigs $(4.7 \pm 1.6 \%$ vs. $19.8 \pm 1.3 \%)$. The position of the measurement of fractional shortening in the LAD area and the LCX

Table II. Coronary Blood Flow in bFGF-treated and Control Animals

\begin{tabular}{|c|c|c|c|c|c|}
\hline & Flow $_{L A D}$ & Flow $_{L C x}$ & Flow $_{\mathrm{LCX}} / \mathrm{Flo}_{\mathrm{LAD}}$ & $\mathbf{R}_{\text {LAD }}$ & $\mathbf{R}_{\mathbf{L C X}}$ \\
\hline \multicolumn{6}{|c|}{ Non-bFGF (control) } \\
\hline Pre-ameroid & NA & NA & $0.83 \pm 0.11$ & NA & NA \\
\hline Prepacing & $1.43 \pm 0.26$ & $1.26 \pm 0.12$ & $0.96 \pm 0.11$ & $1.63 \pm 0.38$ & $1.60 \pm 0.22$ \\
\hline Pacing & $1.96 \pm 0.23$ & $1.53 \pm 0.23$ & $0.80 \pm 0.09 *$ & $0.93 \pm 0.21$ & $1.16 \pm 0.23$ \\
\hline \multicolumn{6}{|c|}{ Basic FGF-Treated } \\
\hline Pre-ameroid & NA & NA & $0.94 \pm 0.06$ & NA & NA \\
\hline Prepacing & $1.36 \pm 0.27$ & $1.23 \pm 0.20$ & $1.00 \pm 0.15$ & $1.45 \pm 0.38$ & $1.41 \pm 0.26$ \\
\hline Pacing & $1.37 \pm 0.21$ & $1.19 \pm 0.15$ & $0.90 \pm 0.07$ & $0.96 \pm 0.18$ & $1.02 \pm 0.07$ \\
\hline
\end{tabular}

Values are mean \pm SE; $F l o w_{L A D}$, myocardial flow in the left anterior descending region $\left(\mathrm{ml} \cdot \mathrm{min}^{-1} \cdot \mathrm{g}^{-1}\right) ; F l o w_{L C X}$, myocardial flow in the circumflex region $\left(\mathrm{ml} \cdot \mathrm{min}^{-1} \cdot \mathrm{g}^{-1}\right) ; R_{L A D}$, coronary resistance in the left anterior descending region $\left(\mathrm{mmHg} / \mathrm{ml} \cdot \mathrm{min}^{-1}\right) ; R_{L C X}$, coronary resistance in the circumflex region $\left(\mathrm{mmHg} / \mathrm{ml} \cdot \mathrm{min}^{-1}\right)$; Pre-ameroid, data before ameroid implantation; $* P<0.05$ compared with prepacing, paired $t$ test. 
Table III. Left Ventricular Hemodynamics in bFGF-treated and Control Animals

\begin{tabular}{|c|c|c|c|c|c|c|}
\hline & HR & LVP & LVEDP & $\% \mathrm{FS}_{\text {LAD }}$ & $\% \mathrm{FS}_{\mathrm{LCX}}$ & Ratio \\
\hline \multicolumn{7}{|c|}{ Non-bFGF (controls) $(n=10)$} \\
\hline Pre-pacing & $105 \pm 8$ & $102 \pm 8$ & $10 \pm 1$ & $19.1 \pm 1.8$ & $3.7 \pm 1.9^{\prime}$ & $0.16 \pm 0.08$ \\
\hline Pacing & $200 \pm 11 *$ & $93 \pm 9 *$ & $24 \pm 4 *$ & $7.1 \pm 1.6^{\ddagger}$ & $0.5 \pm 0.3$ & $0.06 \pm 0.04$ \\
\hline Post-pacing & $125 \pm 8$ & $123 \pm 11$ & $15 \pm 1 *$ & $19.4 \pm 1.3$ & $2.8 \pm 1.4$ & $0.14 \pm 0.07$ \\
\hline $5 \mathrm{~min}$ & $116 \pm 8$ & $110 \pm 9$ & $10 \pm 1$ & $19.1 \pm 1.8$ & $3.7 \pm 1.8$ & $0.18 \pm 0.08$ \\
\hline \multicolumn{7}{|c|}{ Basic FGF-treated $(n=10)$} \\
\hline Pre-pacing & $114 \pm 7$ & $116 \pm 5$ & $10 \pm 1$ & $19.8 \pm 1.3$ & $4.7 \pm 1.6^{9}$ & $0.23 \pm 0.07$ \\
\hline Pacing & $205 \pm 9 *$ & $78 \pm 8^{\ddagger}$ & $17 \pm 3^{*}$ & $4.3 \pm 1.5^{\ddagger}$ & $1.1 \pm 0.6$ & $0.11 \pm 0.08$ \\
\hline Post-pacing & $136 \pm 9$ & $119 \pm 8$ & $11 \pm 1^{\S}$ & $19.8 \pm 1.0$ & $6.0 \pm 1.7$ & $0.31 \pm 0.09 *$ \\
\hline $5 \mathrm{~min}$ & $111 \pm 6$ & $118 \pm 6$ & $9 \pm 1$ & $18.7 \pm 1.1$ & $4.6 \pm 1.6$ & $0.24 \pm 0.08$ \\
\hline
\end{tabular}

Values are mean \pm SE. $L C X$, circumflex region; $L A D$, left anterior descending region; HR, heart rate; $L V P$, left ventricular systolic pressure; $L V E D P$, left ventricular end-diastolic pressure; $\% F S$, percent fractional shortening; Ratio, $\% \mathrm{FS}_{\mathrm{LCX}} / \% \mathrm{FS} \mathrm{LAD}_{\mathrm{L}}{ }^{*} P<0.05,{ }^{\ddagger} P<0.01$ compared with prepacing; ${ }^{8} P<0.05$ compared with non-FGF group; ${ }^{\prime} P<0.05$ compared with $\% \mathrm{FS}_{\mathrm{LAD}}$.

area was fixed on the same trans-axial plane midway between the mitral valve and cardiac apex; therefore, some hearts with large infarctions showed systolic bulging in the examined area. In such cases, we incorporated these data as $0 \%$ fractional shortening. During rapid cardiac pacing, percent fractional shortening fell equally in both LAD and LCX areas in both non-bFGF and bFGF-treated groups. However, while \%FS in the LCX territory fell 7.4-fold in the control group, in bFGF treated animals the \%FS declined only 4.3-fold. Furthermore, there was better recovery in the LCX area after rapid pacing in bFGFtreated than in non-bFGF pigs $(6.0 \pm 1.7 \%$ vs. $2.8 \pm 1.4 \%, P$ $<0.05)$. This is shown more clearly if we normalize the percent fractional shortening in the LCX area $\left(\% \mathrm{FS}_{\mathrm{LCX}}\right)$ by that in the $\mathrm{LAD}$ area $\left(\% \mathrm{FS}_{\mathrm{LAD}}\right)$ as the ratio $\% \mathrm{FS}_{\mathrm{LCX}} / \% \mathrm{FS}_{\mathrm{LAD}}$ (Table III). Also, during pacing the normalized percent fractional shortening in the LCX area tended to be better in bFGF pig (Table III).

LV end-diastolic pressure prior to pacing was equal in both groups. Rapid pacing induced significant LVEDP rise in both groups (Table III). However, while LVEDP remained elevated in the control animals after pacing termination, it promptly returned to normal in bFGF-treated animals. These results show that bFGF administration is able to partially reverse hemodynamic deterioration in the ameroid-compromised territory during pacing, suggesting that bFGF-induced improvement in relative coronary circulation in the LCX territory is of sufficient magnitude to result in functional improvement in the compromised region of the myocardium.

Transthoracic echocardiography. Echocardiographic analysis showed no significant difference in calculated global LV ejection fraction $(45 \pm 4 \%$ vs. $48 \pm 4 \%$, control $(n=6)$ vs. bFGF $(n=7), P=\mathrm{NS})$ or fractional shortening $(0.20 \pm 0.02$ vs. $0.27 \pm 0.03$, control vs. bFGF, $P=\mathrm{NS}$ ), and confirmed increase in anterior wall thickness noted during pathological examination in bFGF-treated pigs $(0.81 \pm 0.04 \mathrm{~cm}$ vs. $0.64 \pm 0.05 \mathrm{~cm}$, bFGF vs. controls, $P<0.05$ ) compared to controls and showed a trend toward higher posterior wall thickness $(0.56 \pm 0.08 \mathrm{~cm}$ vs. $0.48 \pm 0.03 \mathrm{~cm}$, bFGF vs. controls, $P=\mathrm{NS}$ ).

\section{Discussion}

Fibroblast growth factors are pleiotropic molecules capable of stimulating growth of variety of cell types. They are mitogenic and chemotactic for endothelial cells and stimulate endothelial cell production of plasminogen activator protease and collagenase capable of digesting extracellular matrix (23). The FGFs are also mitogenic for smooth muscle (24) as well as a number of other cells (25). Basic FGF is present in sarcoplasma of embryonic cardiac myocytes from the earliest stages of avian heart development (26) and bFGF protein is detectable in both atrial and ventricular adult myocardium as well as in intramyocardial vessels (27). In the developing chicken heart, bFGF expression is limited to the cytoplasm of myocytes: no expression is noted in endothelial or vascular smooth muscle cells. The growth factor potential for stimulating angiogenesis is suggested by the observation that its receptor is expressed in proliferating endothelial and smooth muscle cells after arterial injury (28).

Basic FGF can induce neovascularization in vitro in three dimensional collagen matrix gels (29) as well as in vivo systems such as corneal implants (30) and skin wounds (31). It is involved in a number of disease processes including neovascularization, oncogenesis, and vascular restenosis (32-35), and it may also have endocrine, neurotropic, or vasomotor effects (32). Due to its unique properties, bFGF can be stored in basement membrane and then released by administration of heparin or enzymatic matrix degradation (36-38). Basic FGF-stimulated angiogenesis is said to require an ischemic milieu $(39,40)$, suggesting that the growth factor may be interacting with a variety of other mitogens to achieve this effect and that ischemic conditions may result in upregulation of FGF receptor expression. In particular, transforming growth factor (TGF) beta (41), interferon alpha and IL-2 (42) as well as platelet derived growth factor (PDGF) (43) are known to affect bFGF angiogenic properties. The interplay between bFGF and TGF- $\beta$ is thought to be important in proteolysis (41) and bFGF is known to increase the PDGF- $\alpha$ receptor messenger RNA levels, thereby potentiating PDGF effect on smooth muscle cell proliferation (43). Angiogenesis induced by interferon alpha and IL-2 is also thought to be mediated by bFGF (42).

In vivo administration of bFGF leads to angiogenesis that has been demonstrated in a number of models $(30,39,40,44)$, and the growth factor's in vivo angiogenic potential is thought to be closely coupled with its mitogenic potential (intimal hyperplasia) (2). There is little direct information, however, regarding the role that FGFs play in myocardial angiogenesis. A recent 
report (8) suggests that bFGF injection administered at the time of abrupt coronary artery closure limits infarct size. However, the observed effect may have been more likely due to hemodynamic consequences of an infusion of a large dose of bFGF (known to produce substantial hypotension (45) then its angiogenic properties. We undertook the present study in order to assess bFGF capacity to alleviate chronic myocardial ischemia by stimulating angiogenesis.

We chose to use a periadventitial rather than intracoronary delivery of bFGF because of the ability of polymeric controlled release devices to deliver bFGF to the extravascular space without transendothelial transport and because of the reported block of bFGF effects by functional endothelium (2). On the other hand, intravascular administration appears much less efficient. Thus, intravenously injected bFGF has a half-life of $<3 \mathrm{~min}$ in circulation $(20,46)$ and the growth factor's deposition within arterial wall is almost 40 times less than what is seen with periadventitial delivery (20). Even continuous bFGF infusion did not increase $\left[{ }^{3} \mathrm{H}\right]$ thymidine incorporation in endothelial or smooth muscle cells in rats (46). Furthermore, a recent study using direct coronary infusion of bFGF in a dog ameroid constrictor model failed to show increased angiogenesis associated with growth factor delivery (47). Incorporation of bFGF within the alginate beads allows for prolonged release with first-order release kinetics (2), with retention of $90.2 \pm 3.6 \%$ of the biological activity of the released growth factor. It is estimated that during the linear portion of the release curve the implanted capsule releases $\sim 34 \mathrm{ng} / \mathrm{d}$ to the arterial wall over the course of the $14 \mathrm{~d}(2)$. Furthermore, the segment of the artery proximal to the bFGF-releasing microsphere had half as much growth factor as was deposited within the distal segment. Basic FGF pools around an artery after release from the controlled delivery device. Arterial capillaries and vasa vasorum may absorb the grewth factor, but the marked dilutional effect of a large volume of rapidly flowing blood seen with intravascular administration, is absent. Capillary uptake and other phenomena may in fact expedite transport of the growth factor throughout the wall and to distant vascular sites. Such a mode of bFGF device may also be applicable to clinical settings. Basic FGF-containing capsules are easily attached to tissue, and are held safely for a long time with minimal tissue damage. Although an open-chest operation is needed for placement of this delivery system, nongraftable myocardial areas at risk might be treated in this way during coronary artery bypass graft surgery.

Using a heparin-alginate bead periadventitial delivery system, we found that bFGF administration resulted in fourfold reduction in myocardial infarction mass. Although there was no significant change in distribution of transmural vs. nontransmural infarcts, the reduction in total infarct mass suggests that bFGF improved perfusion of the infarct border zone. To confirm the significance of these changes in myocardial infarct size, we analyzed changes in regional coronary flow as well as hemodynamics in bFGF-treated and controls animals. We found that under baseline conditions there was little difference in LCX coronary flow between both groups. However, regional myocardial blood flow in the LCX territory compared with the blood flow in the LAD territory decreased during rapid pacing in control but not bFGF-treated pigs, thus suggesting better collateral reserve in the bFGF-treated animals. This observation is similar to that of White et al. (14) who showed using an ameroid constrictor model that a deficiency in collateral circulation was unmasked by adenosine administration. In a previous study using the same ameroid constrictor model (19), we demonstrated that bFGF administration restores receptor-mediated endothelial function in the chronically ischemic region. This improvement in endothelial function may in part explain the improved coronary reserve seen in bFGF-treated animals.

It is conceivable, although highly unlikely, that baseline differences in the amount of collateral flow between the group produced the observed differences in the LCX flow and infarct size seen in the study. We did not detect any differences in the baseline flow in the LCX territory at the time of ameroid placement or at the time of follow up study (see Table II). Indeed, the differences in coronary flow between the groups were observed only during rapid pacing suggesting that collateral recruitment in bFGF-treated animals was responsible for the observed effect.

It is interesting to note that bFGF-treated pigs while demonstrating a significant drop in coronary resistance during rapid pacing, did not show an increase in the total (LCX + LAD) coronary flow. This observation is probably due to a relatively larger drop in arterial pressures seen during pacing in bFGFtreated animals than in controls with a lower systemic pressure limiting increase in coronary flow and not a specific effect of bFGF on the coronary vessels. The observed decline in coronary resistance noted in both control and bFGF groups (see Table II), expected with pacing further demonstrates appropriate response of coronary vasculature in both animal groups.

We next analyzed the effect of bFGF administration on global and regional myocardial function. We found no significant differences in baseline hemodynamic parameters (heart rate, blood pressure, LVEDP, regional fractional shortening, and ejection fraction) between both groups at rest, despite the differences in the infarct size. One possible explanation is that the LAD area (nonischemic area) may adequately compensate for the dysfunction of the LCX area (ischemic area). During rapid cardiac pacing, both groups of animals showed increases in LVEDP, a fall in systemic blood pressure, and a decline in fractional shortening in both LAD and LCX territories. However, the LVEDP rise in bFGF group was less than in controls ( 7 vs. $14 \mathrm{~mm} \mathrm{Hg}$ ). At the same time, bFGF-treated animals showed significantly better preservation of fractional shortening in the LCX territory during pacing as well as better post-pacing recovery than controls. These hemodynamic observations, taken together with coronary flow findings, suggest that bFGF administration used in this study resulted in functionally significant improvement in collateral myocardial circulation to the chronically ischemic zone.

It is also interesting to note that bFGF administration resulted in the increased thickness of both LV anterior and posterior walls demonstrated morphologically as well as echocardiographically. While there was some disagreement between the techniques (primarily due to echocardiographic imprecisions in measurement of posterior wall thickness), there was an overall correlation between both sets of data $\left(r^{2}=0.49, P=0.02\right.$, data not shown). The increase in ventricular wall thickness further correlated with increased LV mass seen in bFGF-treated animals $\left(92.5 \pm 4.4 \mathrm{~cm}^{3}\right.$ vs. $74.0 \pm 3.5 \mathrm{~cm}^{3}$, bFGF vs. controls, $P$ $<0.01)$. However, there was no difference in body weights between the two groups of animals (see Table I) suggesting that increase in left ventricular mass was due to specific cardiac events and not to the difference in animal size. A number of process could result in increased wall thickness, and, consequently, left ventricular mass. Basic FGF has been shown to induce proliferation of a number of cell types in porcine myocardium. Thus, thymidine labeling experiments performed on pigs 
after ameroid constrictor placement, demonstrate highest incorporation $3 \mathrm{wk}$ after implantation (time that corresponds to approximately $3 \mathrm{~d}$ after vessel occlusion) with above background tritium labeling present for up to $8 \mathrm{wk}$ after ameroid implantation. Endothelial cells have the most frequently labeled nuclei followed by adventitial fibroblasts and smooth muscle cells (48). Alternatively, increased matrix production associated with neovascularization could also potentially accounted for the increased wall thickness and LV mass. Finally, accumulation of blood in newly formed vessels could have led to myocardial engorgement similar to an erectile effect seen in left ventricular hypertrophy (49), resulting in increased wall thickness and heart weight. The exploration of these possibilities will require further investigations.

Limitations of the study. In the present investigation we analyzed the effect of periadventitial delivery of bFGF initiated simultaneously with the implantation of an ameroid constrictor, on myocardial function in pigs. Although we found a number of beneficial effects of treatment, a number of issues remain to be addressed. While it seems likely that beneficial effect of bFGF administration results from increased angiogenesis as demonstrated by increased coronary flow and reduced infarct size, it is conceivable, although unlikely, that the observed differences resulted from delayed closure of ameroid constrictor in bFGF-treated animals due to local vasadilatory effects of bFGF as well as its ability to induce nitric oxide release. It should be noted however, that concentrations of bFGF necessary for these effects are several order of magnitude larger than those used in the present study (19). Furthermore, it is possible that baseline differences in the degree of preexisting collateral circulation between the two groups of animals resulted in the effects seen in the bFGF group. Although we did not systematically measure baseline collateral flow in all animals, we did not detect any substantial degree of collateral circulation in several animal studied, a result in accord with the notion that normal porcine hearts possess little collateral circulation (48).

It is not clear what is the optimal dose of bFGF or the length or route of administration. Furthermore, bFGF may not be the best growth factor in these settings and other mitogens such as acidic FGF (50), vascular endothelial growth factor (51), or TGF- $\beta$ (41), to name just a few, might be preferred (52). Alternatively, a combination of several growth factors may provide optimal benefit. It is also important to note that in the usual clinical setting, one is faced with myocardium that is already ischemic. The present study doesn't address the effectiveness of bFGF under such conditions. Finally, intracoronary delivery of an effective angiogenic factor would be preferable to periadventitial administration in that it would allow application by the catheter-based technology and therefore not require openchest surgery. These and other issues are the subject of current investigations.

\section{Acknowledgments}

We would like to thank Dr. Boris Nunez for his help with coronary angiography and Pat Allen and John Juster for expert secretarial assistance.

This work was supported by an American Heart Association Clinician-Scientist Award 9100420 (M. Simons), grant HL-46716 (F. W. Sellke) and HL-44431 (W. Grossman) from the National Heart, Lung, and Blood Institute, American Heart Association-Massachusetts affiliate grant 13-501-912 (F. W. Sellke), a Physician-Scientist Award from the American College of Chest Physicians (F. W. Sellke), a Physician-
Scientist Award (E. R. Edelman) from the National Institute of Health (K12 AG00294), and the Edward Mallinckrodt, Jr. Foundation (W. J. Manning).

\section{References}

1. Linder, V., R. A. Majack, and M. A. Reidy. 1990. Basic fibroblast growth factor stimulates endothelial regrowth and proliferation in denuded arteries. $J$. Clin. Invest. 85:2004-2008.

2. Edelman, E. R., M. A. Nugent, and M. J. Karnovsky. 1992. Basic fibroblast growth factor enhances the coupling of intimal hyperplasia and proliferation of vasa vasorum in injured rat arteries. J. Clin. Invest. 89:465-473.

3. Linder, V., D. A. Lappi, A. Baird, R. A. Majack, and M. A. Reidy. 1991. Role of basic fibroblast growth factor in vascular lesion formation. Circ. Res 68:106-113.

4. Montesano, R., J. D. Vassali, R. Baird, R. Guillemin, and L. Orci. 1986. Basic fibroblast growth factor induces angiogenesis in vitro. Proc. Natl. Acad Sci. USA. 83:7297-7301.

5. Connolly, D. T., B. L. Stoddard, N. K. Harakas, and J. Feder. 1987. Human fibroblast growth factor is a mitogen and chemoattractant for endothelial cells. Biochem. Biophys. Res. Commun. 144:705-712.

6. Presta, M., J. A. M. Maier, M. Rusnati, and G. Ragnotti. 1989. Basic fibroblast growth factor is released from endothelial extracellular matrix in a biologically active form. J. Cell. Physiol. 140:68-74.

7. Schweigere, L., G. Neufeld, J. Friedman, J. A. Abraham, J. C. Fiddes, and D Gospodarowicz. 1987. Capillary endothelial cells express basic fibroblast growth factor, a mitogen that promotes their own growth. Nature (Lond.). 325:257-259.

8. Yanagisawa-Miwa, A., Y. Uchida, F. Nakamura, T. Tomaru, H. Kido, T. Kamijo, T. Sugimoto, K. Kaji, M. Utsuyama, C. Kurashima, and H. Ito. 1992. Salvage of infarct myocardium by angiogenic action of basic fibroblast growth factor. Science (Wash. DC). 257:1401-1403.

9. Casscells, W., E. Speir, J. Sasse, M. Klagsbrun, P. Allen, M. Lee, B. Calvo, M. Chiba, L. Haggroth, J. Folkman, and S. E. Epstein. 1990. Isolation, characterization, and localization of heparin-binding growth factor in the heart. J. Clin. Invest. 85:433-441.

10. Heusch, G., B. D. Guth, R. Steitelberger, and J. Ross, Jr. 1987. Recruitment of coronary vasodilator reserve by nifedipine attenuates exercise-induced myocardial ischemia in dogs. Circulation. 75:482-490.

11. White, F. C., M. D. McKirnan, E. A. Breisch, B. D. Guth, M. Liu, and C. M. Bloor. 1981. Coronary collateral circulation in the pig: correlation of collateral flow and coronary bed. Basic Res. Cardiol. 76:189-196.

12. Roth, D. M., Y. Maruoka, J. Rogers, F. C. White, J. C. Longhurst, and C. M. Bloor. 1987. Development of coronary collateral circulation in left circumflex Ameroid-occluded swine myocardium. Am. J. Physiol. 253:H1279-H1288.

13. Sellke, F. W., J. E. Quillen, L. A. Brooks, and D. G. Harrison. 1990 Endothelial modulation of the coronary vasculature in vessels perfused via mature collaterals. Circulation. 81:1938-1947.

14. White, F. C., S. M. Carroll, A. Magnet, and C. M. Bloor. 1992. Coronary collateral development in swine after coronary artery occlusion. Circ. Res. 71:1490-1500.

15. Roth, D. M., F. C. White, O. Mathieu-Costello, B. D. Guth, G. Heusch, C. M. Bloor, and J. C. Longhurst. 1987. Effect of left circumflex ameroid constrictor placement on adrenergic innervation of myocardium. Am. J. Physiol. 253:H1425-H1434.

16. O'Konski, M. S., F. C. White, J. C. Longhurst, D. M. Roth, and C. M. Bloor. 1987. Ameroid constriction of the proximal left circumflex coronary artery in swine. Am. J. Cardiovasc. Pathol. 1:69-77.

17. White, F. C., D. M. Roth, and C. M. Bloor. 1989. Coronary collateral reserve during exercise induced ischemia in the pig. Basic. Res. Cardiol. 84:4254.

18. Sellke, F. W., Y. Kagaya, R. G. Johnson, T. Shafique, F. J. Schoen, W. Grossman, and R. M. Weintraub. 1992. Endothelial modulation of porcine coronary microcirculation perfused via immature collaterals. Am. J. Physiol. 262(Heart Circ Physiol 31):H1669-H1675.

19. Sellke, F. W., S. Y. Wang, K. Harada, M. Friedman, E. R. Edelman, W. Grossman, and M. Simons. 1994. Basic fibroblast growth factor enhances endothelium-dependent relaxation of the collateral-perfused porcine coronary microcirculation. Am. J. Physiol. In press.

20. Edelman, E. R., M. A. Nugent, and M. J. Karnovsky. 1993. Perivascular and intravenous administration of basic fibroblast growth factor: Vascular and solid organ deposition. Proc. Natl. Acad. Sci. USA. 90:1513-1517.

21. Edelman, E. R., E. Mathiowitz, R. Langer, and M. Klagsbrun. 1991. Controlled and modulated release of basic fibroblast growth factor. Biomaterials. 12:619-626.

22. Kowallik, P., R. Schulz, B. D. Guth, A. Schade, W. Paffhausen, R. Gross, and G. Heusch. 1991. Measurement of regional myocardial blood flow with multiple colored microspheres. Circulation. 83:974-982.

23. Burgess, W. H., and T. Maciag. 1989. The heparin-binding (fibroblast) growth factor family of proteins. Annu. Rev. Biochem. 58:575-606. 
24. Ledoux, D., L. Gannoun-Zaki, and D. Barritault. 1992. Interactions of FGFs with target cells. Prog. Growth Factor Res. 4:107-120.

25. Folkman, J., and Y. Shing. 1992. Angiogenesis. J. Biol. Chem. 267:10931 10934.

26. Parlow, M. H., D. L. Bolender, N. P. Kokan-Moore, and J. Lough. 1991. Localization of bFGF-like proteins as puctuate inclusions in the preseptation myocardium of the chicken embryo. Dev. Biol. 146:139-147.

27. Kardami, E., and R. R. Fandrich. 1989. Basic fibroblast growth factor in atria and ventricles of the vertebrate heart. J. Cell Biol. 109:1865-1875.

28. Lindner, V., and M. A. Reidy. 1993. Expression of basic fibroblast growth factor and its receptor by smooth muscle cells and endothelium in injured rat arteries. An en face study. Circ. Res. 73:589-595.

29. Sakuda, H., Y. Nakashima, S. Kuriyama, and K. Sueishi. 1992. Media conditioned by smooth muscle cells cultured in a variety of hypoxic environments stimulates in vitro angiogenesis. Am. J. Pathol. 141:1507-1616.

30. Gaudric, A., T. N'guyen, M. Moenner, A. Glacet-Bernard, and D. Barritault. 1992. Quantification of angiogenesis due to basic fibroblast growth factor in a modified rabbit corneal model. Ophthalmic Res. 24:181-188.

31. Mellin, T. N., R. J. Mennie, D. E. Cashen, J. J. Ronan, J. Capparella

M. L. James, J. Disalvo, J. Frank, D. Linemeyer, G. Gimenez-Gallego, and K. A Thomas. 1992. Acidic fibroblast growth factor accelerates dermal wound healing. Growth Factors. 7:1-14.

32. Gospodarowicz, D. 1991. Fibroblast growth factors: from genes to clinical applications. Cell. Biol. Rev. 25:307-16, 337-339.

33. Villaschi, S., and R. F. Nicosia. 1993. Angiogenic role of endogenous basic fibroblast growth factor released by rat aorta after injury. Am. J. Pathol. 143:181-190.

34. Lindner, V., and M. A. Reidy. 1991. Proliferation of smooth muscle cells after vascular injury is inhibited by an antibody against basic fibroblast growth factor. Proc. Natl. Acad. Sci. USA. 88:3739-3743.

35. Morrison, R. S. 1991. Suppression of basic fibroblast growth factor expression by antisense oligodeoxynucleotides inhibits the growth of transformed human astrocytes. J. Biol. Chem. 266:728-734.

36. Vlodavsky, I., J. Folkman, R. Sullivan, R. Fridman, R. Ishai-Michaeli, J. Sasse, and M. Klagsbrun. 1987. Endothelial cell-derived basic fibroblast growth factor: synthesis and deposition into subendothelial extracellular matrix. Proc. Natl. Acad. Sci. USA. 84:2292-2296.

37. Folkman, J., M. Klagsbrun, J. Sasse, M. Wadzinski, D. Ingber, and I. Vlodavsky. 1988. A heparin-binding angiogenic protein-basic fibroblast growth factor-is stored within basement membrane. Am. J. Pathol. 130:393-400.

38. Klagsbrun, M. 1992. Mediators of angiogenesis: the biological significance of basic fibroblast growth factor (bFGF)-heparin and heparan sulfate interactions. Semin. Cancer Biol. 3(2):81-87.

39. Baffour, R., J. Berman, J. L. Garb, S. W. Rhee, J. Kaufman, and P. Friedmann. 1992. Enhanced angiogenesis and growth of collaterals by in vivo administration of recombinant basic fibroblast growth factor in a rabbit model of acute lower limb ischemia: dose-response effect of basic fibroblast growth factor. J. Vasc. Surg. 16:181-191

40. Chleboun, J. O., R. N. Martins, C. A. Mitchell, and T. V. Chirila. 1992. bFGF enhances the development of the collateral circulation after acute arterial occlusion. Biochem. Biophys. Res. Commun. 185:510-516.

41. Pepper, M. S., D. Belin, R. Montesano, L. Orci, and J. D. Vassalli. 1990. Transforming growth factor-beta 1 modulates basic fibroblast growth factorinduced proteolytic and angiogenic properties of endothelial cells in vitro. J. Cell Biol. 111:743-755.

42. Cozzolino, F., M. Torcia, M. Lucibello, L. Morbidelli, M. Ziche, J. Platt, S. Fabiani, J. Brett, and D. Stern. 1993. Interferon-alpha and interleukin 2 synergistically enhance basic fibroblast growth factor synthesis and induce release, promoting endothelial cell growth. J. Clin. Invest. 91:2504-2512.

43. Schullmann, C., R. Grugel, D. Tatje, J. Hoppe, J. Folkman, D. Marm, and H. A. Weich. 1992. Basic fibroblast growth factor modulates the mitogenic potency of the platelet-derived growth factor (PDGF) isoforms by specific upregulation of the PDGF $\alpha$ receptor in vascular smooth muscle cells. J. Biol. Chem. 267:18032-18039.

44. Cuevas, P., G. Gimenez-Gallego, F. Carceller, B. Cuevas, and A. Crespo. 1993. Single topical application of human recombinant basic fibroblast growth factor promotes neovascularization in rat cerebral cortex. Surg. Neurol. 39:380384

45. Cuevas, P., F. Carceller, S. Ortega, M. Zazo, I. Nieto, and G. GimenezGallego. 1991. Hypotensive activity of fibroblast growth factor. Science (Wash. DC). 254:1208-1210.

46. Whalen, G. F., Y. Shing, and J. Folkman. 1989. The fate of intravenously administered bFGF and the effect of heparin. Growth Factors. 1:157-164.

47. Unger, E. F., S. Banai, M. Shou, M. Jaklitch, E. Hodge, R. Correa, M. Jaye, and S. E. Epstein. 1993. A model to assess interventions to improve collateral blood flow: continuous administration of agents into the left coronary artery in dogs. Cardiovasc. Res. 27:785-791.

48. Schaper, W., H. S. Sharma, W. Quinkler, T. Markert, M. Wunsch, and J. Schaper. 1990. Molecular biologic concepts of coronary anastamoses. J. Am. Coll. Cardiol. 15:513-518.

49. Momomura, S., J. J. Ferguson, M. J. Miller, J. A. Parker, and W. Grossman. 1991. Regional myocardial blood flow and left ventricular diastolic properties in pacing-induced ischemia. J. Am. Coll. Cardiol. 17:781-789.

50. Nabel, E. G., Z. Y. Yang, G. Plautz, R. Forough, X. Zhan, C. C. Haudenschild, T. Maciag, and G. J. Nabel. 1993. Recombinant fibroblast growth factor-1 promotes intimal hyperplasia and angiogenesis in arteries in vivo. Nature (Lond.). 362:844-846.

51. Millauer, B., S. Wizigmann-Voos, H. Schnurch, R. Martinez, N. P. Moller, W. Risau, and A. Ullrich. 1993. High affinity VEGF binding and developmental expression suggest Flk-1 as a major regulator of vasculogenesis and angiogenesis. Cell. 72:835-846.

52. Schaper, W. 1991. Angiogenesis in the adult heart. Basic Res. Cardiol. 86(Suppl 2):51-56. 